\title{
Fault Diagnosis and Detection in Industrial Motor Network Environment Using Knowledge-Level Modelling Technique
}

\author{
Saud Altaf, ${ }^{1}$ Muhammad Waseem Soomro, ${ }^{2}$ and Mirza Sajid Mehmood ${ }^{3}$ \\ ${ }^{1}$ Sensor Network and Smart Environment Research Centre, Auckland University of Technology, Auckland, New Zealand \\ ${ }^{2}$ School of Professional Engineering, Manukau Institute of Technology, Auckland, New Zealand \\ ${ }^{3}$ Sajid Brothers Engineering Industries (Pvt.) Ltd., Gujranwala, Pakistan \\ Correspondence should be addressed to Saud Altaf; drsaudaltaf@gmail.com
}

Received 24 August 2016; Revised 13 November 2016; Accepted 23 November 2016; Published 9 January 2017

Academic Editor: Dimitrios E. Manolakos

Copyright (C) 2017 Saud Altaf et al. This is an open access article distributed under the Creative Commons Attribution License, which permits unrestricted use, distribution, and reproduction in any medium, provided the original work is properly cited.

\begin{abstract}
In this paper, broken rotor bar (BRB) fault is investigated by utilizing the Motor Current Signature Analysis (MCSA) method. In industrial environment, induction motor is very symmetrical, and it may have obvious electrical signal components at different fault frequencies due to their manufacturing errors, inappropriate motor installation, and other influencing factors. The misalignment experiments revealed that improper motor installation could lead to an unexpected frequency peak, which will affect the motor fault diagnosis process. Furthermore, manufacturing and operating noisy environment could also disturb the motor fault diagnosis process. This paper presents efficient supervised Artificial Neural Network (ANN) learning technique that is able to identify fault type when situation of diagnosis is uncertain. Significant features are taken out from the electric current which are based on the different frequency points and associated amplitude values with fault type. The simulation results showed that the proposed technique was able to diagnose the target fault type. The ANN architecture worked well with selecting of significant number of feature data sets. It seemed that, to the results, accuracy in fault detection with features vector has been achieved through classification performance and confusion error percentage is acceptable between healthy and faulty condition of motor.
\end{abstract}

\section{Introduction}

Induction motors, also named as asynchronous motor, are generally using equipment in manufacturing industry, petrochemical, transportation, and power systems, due to simple structure, high overload capability, reliability, and better efficiency. Different sizes of induction motors are available in industry from small to over $100 \mathrm{~K}$ Horsepower (Hp) [1]. Compared with direct current motors, the induction motors are more rugged, cheap, and require less overall maintenance. Therefore, these are preferred choice as industrial motors by engineers. The rotating parts do not require connecting electricity because electromagnetic induction provides the transfer of energy from stationary parts to the rotating components. The stator produces a rotating magnetic field, which convert into alternating electromotive force and current in the motor rotor. This rotor current and the rotating components of the stationary winding interact with each other and produce a motor torque. The characteristic of torque-speed is related to the components resistance and reactance of the rotor. Therefore, with different percentage values of rotor resistance to reactance in rotor circuits, it is possible to achieve different torque-speed characteristics [2].

Minor symptoms of motor faults may cause lower efficiency, high energy utilization, improper performance, and long-time equipment operating shutdown. Even minor faults can increase the loss chances such as reducing efficiency and increasing motor temperature, which will reduce the winding insulation life span and increasing motor vibration [3]. They are caused by the operating environment circumstances and the equipment internal mechanical factors. Therefore, the diagnosis of motor faults is important task for engineers at early stage and avoids maintenance cost.

Different methods of induction motor fault diagnosis were underinvestigated by few researchers and different techniques have been proposed [4] for fault diagnosis. But, 
the most common used approach is Motor Current Signature Analysis (MCSA) [5]. Several induction motor faults diagnosis techniques are based on Fast Fourier Transform (FFT) using electrical signal signature analysis. Other diagnosis methods including vibration analysis, temperature measurements, harmonic analysis of speed fluctuations, vibration monitoring, state and parameters estimation, either axial flux, acoustic noise measurement, and magnetic field analysis [6] may diagnose through other techniques, for example, ShortTime Fourier Transform (STFT) and Wavelet. Currently, Artificial Intelligence (AI) techniques have been combined with traditional diagnosis methods for detection of the right faults, such as Fuzzy Logic (FL), Genetic Algorithms (GA), Neural Network (NN), Bayesian classifiers, and envelope technique [7].

The methods used to diagnose BRB fault can be generally categorized into two types, invasive and noninvasive fault diagnosis techniques [8]. The invasive methods diagnose the broken bars by monitoring the deviation of the magnetic potential vector and asymmetrical magnetic flux distribution, gyration radius, asymmetrical magnetic flux distribution, torque, and speed fluctuation. In this method, sensors and costly measurement equipment are needed, which will make the process of the diagnostic method more complex and expensive.

Induction motor faults often generate particular frequency components in the electric current spectrum. The abnormal harmonics contain potential information of motor faults. Therefore, the frequency analysis approach is the most commonly used method to diagnose induction motor faults.

MCSA is commonly used method for diagnosing the motor faults due to its simple implementation and low cost [8]. This technique is based on the detection of sidebands around the fundamental frequency in the stator electric current signal [9]. Firstly, the motor faults are simulated to observe the spectrum of stator currents for each fault condition. From the simulation, abnormal harmonics of stator currents are obtained as reference signals. Secondly, the recorded stator current signal is transferred from time domain to frequency domain by using Fourier Transform. Finally, the motor faults could be diagnosed through the comparison of recorded stator current signals and the reference signals in the frequency domain.

Some latest research has investigated fault diagnosis process that focused on broken rotor fault detection at various load level. In [10], Naha et al's MCSA technique is used to detect the BRB fault under low-level load condition using the combination of FFT and Hilbert Transform (HT) on small induction motors. But only half part of the fault signal around the supply frequency was considered without any knowledge-level approach for decision-making. The Kalman filter is applied to identify and estimate the fundamental frequency components of the stator current spectrum and removal of noisy signal. But, through this method, some significant features are also removed from the signal that plays an important role in decision-making if knowledgelevel technique is used in parallel. Climente-Alarcon et al. [11] discussed the injection method of high frequency noisy signal into healthy signal to create the fault abnormal components at standstill operating condition. But, this method is not suitable when the induction motor is operating with a variable frequency drive at low-load level. And it also requires additional experimental setup arrangement and cost as well.

On the other hand, one can observe a potential growing interest in fault diagnosis process using different knowledgelevel techniques. Many research studies have investigated the field of fault detection using isolated induction motors based on diffident AI technique [12-15]. The Artificial Neural Network (ANN) has been perhaps the most commonly used artificial intelligence technique in motor condition monitoring and fault diagnosis due to its excellent pattern recognition ability and ability to recognize fuzzy and indefinite signals. Eldin et al. [16] presented a diagnosis system based on ANN on machines isolated from system, which applies the RMS measurements of current, voltage, and speed to train the ANN in diagnosis of motor rotor faults. Voltage faults are only identified in a steady-state condition, not in a dynamicload condition. Another study was presented by Arabac1 and Bilgin [17], based on the influence of the rotor fault on current in the frequency domain, using ANN in a steadymotor operating condition. This study demonstrated the possible symptoms of significant frequency components on the frequency spectrum related to a broken rotor bar fault. These symptoms are used as an input matrix using the supervised ANN architecture. But the selection criteria of hidden layer were not addressed in that condition when the signal characteristics are in steady state. The proposed technique concluded that the process of rotor fault diagnosis and discrimination between each fault occurred with reasonable accuracy.

This paper presents the industrial experimental setup for fault diagnosis process and tested modelled multiple ANN architectures for decision-making. In this research, fault diagnosis is hypothesized as a pattern recognition and classification problem that contains the association of different patterns of input data sets, demonstrating the behaviour of the motor toward fault conditions. A significant number of feature samples are extracted from the current which is based on the different frequency points and associated amplitude values with each fault type. Various types of neural network architectures are tested for the training to adjust the weights of hidden layer until targeted output is achieved for satisfactory performance.

\section{Broken Rotor Bar Fault Detection Method}

The frequency components that are related to a broken rotor bar (BRB) condition for slips [18] are

$$
f_{\mathrm{BRB}}=(1 \pm 2 k s) f_{1} \text {, }
$$

where $k=1,2,3, \ldots$ and $f_{1}$ is fundamental frequency.

Major BRB frequencies $(k=1)$ may appeared very close to the fundamental supply frequency $f_{1}$ and incorporated with the low-level signal ratio with noise that makes the task of detecting the BRB condition difficult. However, if the load torque depends on time, then the spectral estimation should be done using a very short data window. Methods based on 
the Discrete Fourier Transform (DFT) are not able to detect frequencies close to the fundamental when using a very short data window. In this situation, Fast Fourier Transform (FFT) is the suitable technique that overcomes the time-dependent issue [18].

\section{Feature Selection and Extraction}

In this research, we have chosen six significant features in diagnosing the targeted faults at each motor level. The synchronized speed of each motor, the rotor slip value, the amplitude value of each fault frequency component, the frequency Root Mean Square (RMS), and the crest factor value are preferred. The reason for considering the speed and slip of each motor as a feature is because these two parameters are associated with BRB and eccentricity faults. Without these values, target faults are difficult to diagnose in motor fault in running mode. Motor slip has been presented with different notations for each fault to estimate the associated synchronized speed at each individual significant frequency sideband point. The amplitude values of each fault frequency component show the severity of suspected frequency points for BRB and eccentricity faults. Finally, the RMS and crest factor features were chosen to identify the noise signals or other unknown fault symptoms.

In this paper, MATLAB/Simulink is used to extract the features values through model and script. The Root Mean Square (RMS), synchronized speed, rotor slip, and crest factor of the motor are the data that provides the information on motor operating condition. In order to calculate the significant value for each of the features from each motor data regarding operational condition, the following equations (2) are formulated as given [19]:

$$
\begin{gathered}
\text { Speed }=x_{1}=S_{\mathrm{rpm}}=\frac{120 \times F}{P}, \\
\text { Slip }=x_{2}=S=\frac{n_{s}-n_{r}}{n_{s}} \times 100 \%, \\
\text { RMS }=x_{3}=X_{\mathrm{RMS}}=\sqrt{\frac{1}{n} \sum_{i=1}^{n} x_{i}^{2},} \\
\text { CrestFactor }=x_{4}=\frac{\mathrm{Amp}_{\max }}{X_{\mathrm{RMS}}} .
\end{gathered}
$$

\section{Experiment Setup for Faults Diagnosis}

For the development of test bed environment, a noisy motor operating environment is chosen for experiment where other equipment is also running in parallel. The experimental setup and motor parameters are presented in Figure 1 and Table 1, respectively. The fault is created in motor artificially by damaging the bars in 3-phase induction motor. For the online test, an oscilloscope is used to record the current signal data through current probe and capture the current signal and store in USB drive for analysis. The signal is analysed by using MATLAB/Simulink model. For the creation of complexity in analysis, two types of load levels (no load and full load)

\begin{tabular}{|c|c|}
\hline $\begin{array}{l}\text { Amplitude } \\
\text { difference }(-\mathrm{dB})\end{array}$ & Rotor condition \\
\hline$>50$ & In excellent condition \\
\hline $44-50$ & Good condition \\
\hline $38-44$ & Moderate condition \\
\hline $32-38$ & $\begin{array}{l}\text { Rotor bar crack may be in initial stage or } \\
\text { high resistance in joints }\end{array}$ \\
\hline $26-32$ & $\begin{array}{l}\text { Two rotor bars may be cracked or high } \\
\text { resistance in joints }\end{array}$ \\
\hline $20-26$ & Several bars cracked or rings end \\
\hline$<20$ & Multiple broken bars \\
\hline
\end{tabular}

TABLE 1: Induction motor parameters.

\begin{tabular}{lc}
\hline Motor parameter & Value \\
\hline Power [kW] & 2.2 \\
Voltage [V] & $380-420$ \\
Current [A] & 4.9 \\
Supply frequency [Hz] & 50 \\
Rotor speed [rpm] & 1430 \\
Poles & 4 \\
\hline
\end{tabular}

TABLE 2: Assessing rotor condition [5].

are applied to observe the abnormal behaviour of current signal because, at no-load level, it is very difficult to identify significant sidebands and decide the condition of motor.

\section{Frequency Analysis Simulation Results}

In order to develop a faults index library, we took the sample data at different torque load condition to observe the sidebands around the supply frequency. Table 2 shows that assessing the rotor bars condition depends on comparing the difference between amplitude value of supply frequency and possible sidebands that appear around the supply frequency $(50 \mathrm{~Hz})$.

For the development of case study, BRB faults are created artificially by damaging the bars. Figures 2-4 show the realtime data capture from motor with different load torque condition in every 5 seconds of interval of time, with a horizontal axis of frequency $(\mathrm{Hz})$ and vertical amplitude $(\mathrm{dB})$ as given by Figures 2, 3, and 4 .

As shown in Figure 2, four samples were taken in different interval of time and there is no significant sideband that appears around the fundamental frequency due to nonlinear nature of the current signal and within no-load condition. Because, it is very difficult to observe the sideband components in spectrum without applying any load. A small sideband appeared at $47 \mathrm{~Hz}$ in Sample 1 and Sample 2 data in negative pole side, but the amplitude value is not showing any type of symptoms of BRB fault. We can consider this small peak on same point as a noise that is manifested into healthy signal from motor operating environment. In Figure 3, two significant sidebands are observed in all samples time with different amplitude, showing the clear symptoms of two broken bars and cracks in rotor as well. Figure 4 presented 


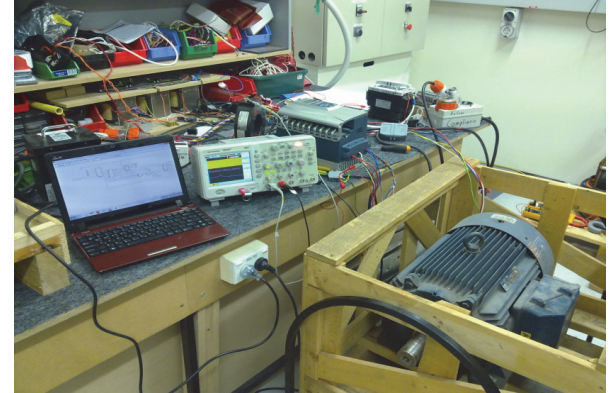

FIgURE 1: The industrial setup for induction motor experiment.



FIGURE 2: Measured current spectrum at no-load condition.

more broken bars symptoms in both pole sides around the fundamental frequency.

Figure 5(a) highlighted the rotor current response and it can be clearly observed that the rotor current value with no load after a certain timeframe was running smoothly and linearly. But once motor was on full-load torque, it shows a dramatic change in rotor current spectrum, presenting the abnormal behavioural influence in current characteristics as shown in Figure 5(b). Due to the uncharacteristic vibrational behaviour of broken bars, the characteristics feature value of torque also are changed and cause changing of the speed of motor and variation in torque level curve, as shown in Figure 6, respectively.

In reference to Figures 2-6, as we can observe from Table 3, at no-load level, the speed of motor is higher than the other two load conditions due to nonresistance. But the slip value is less among others due to nonvariance speed of motor. The sidebands values are not significantly clear, showing the symptoms of BRB fault. However, at full-load condition, the sidebands have considerable amplitude values that represent the motor condition as faulty.

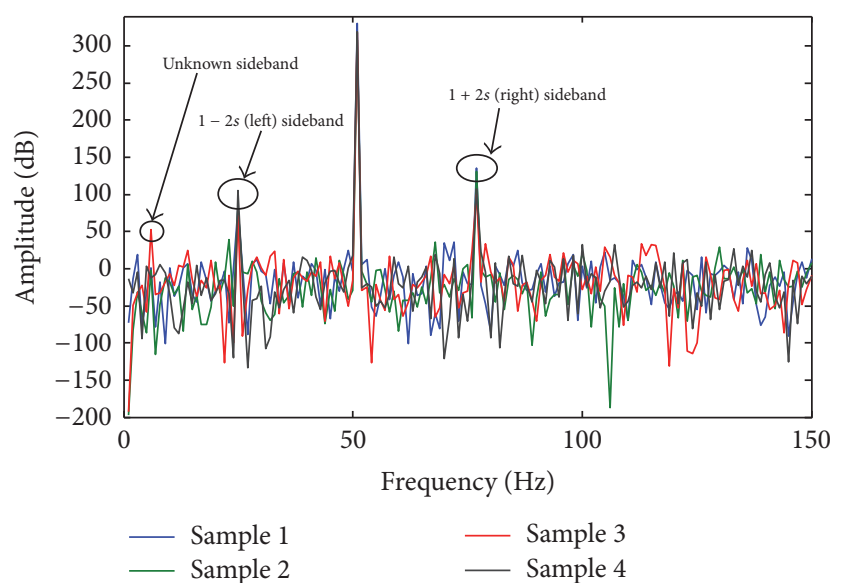

FIGURE 3: Measured current spectrum at full load with two broken rotor bars showing $(1 \pm 2 s) f_{s}$ sidebands.

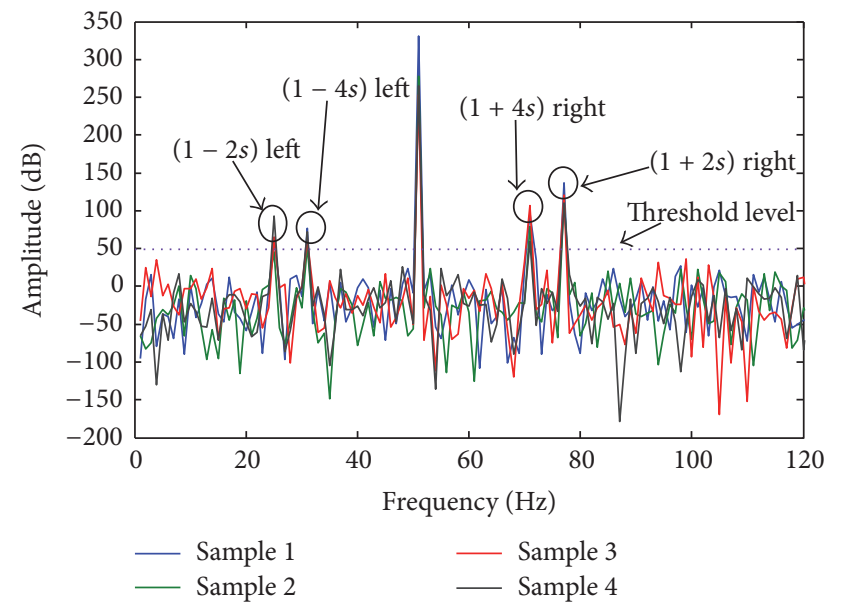

FIGURE 4: Measured current spectrum around the $50 \mathrm{~Hz}$ peak for a motor with more broken rotor bar and crack showing $(1 \pm 2 s) f_{s}$ and $(1 \pm 4 s) f_{s}$ sidebands in both poles.

\section{Neural Network Architecture Modelling to Avoid Uncertainty Management}

In the form of transform signal, sometimes, it may be complex to apply the traditional mathematical technique in fault diagnosis process. While Feed Forward Neural Network (FFNN) technique has been allowing input/output mapping that will be employed between the nodes with nonlinear relationship [19], neural network has ability to recognize the abnormal representation of electrical signal due to their inherent capacity of classification and generalization process, especially, when response time and sensitivity of the actual process presented the repetition of fault sets and created the uncertainty in fault identification in power network. Several training algorithms have been proposed, but BPNN algorithm is most commonly used in for isolated machine faults [20].

A multilayer FFNN is used in this research for type identification and localization of fault within network. For 


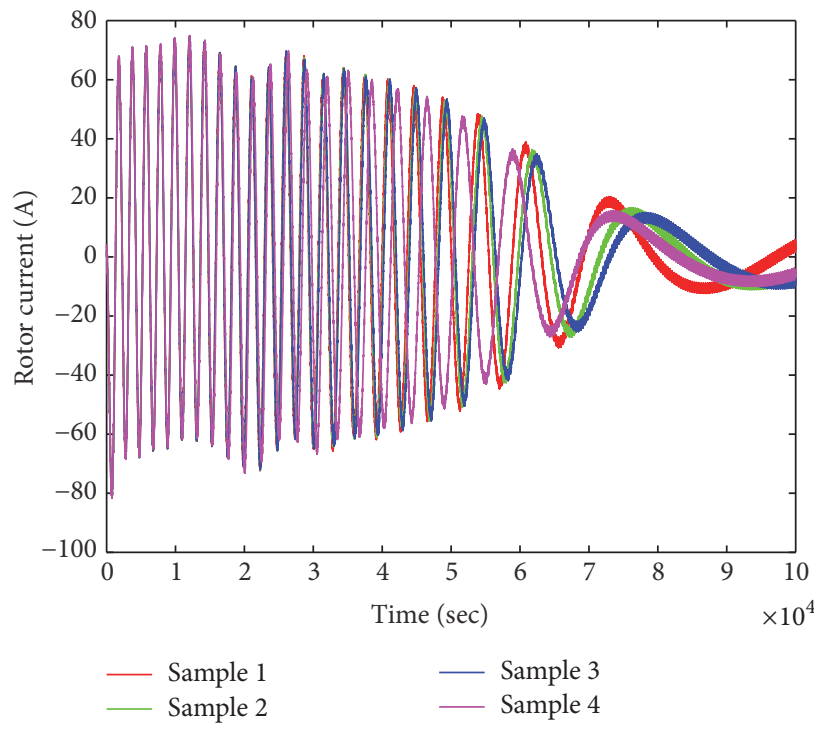

(a)

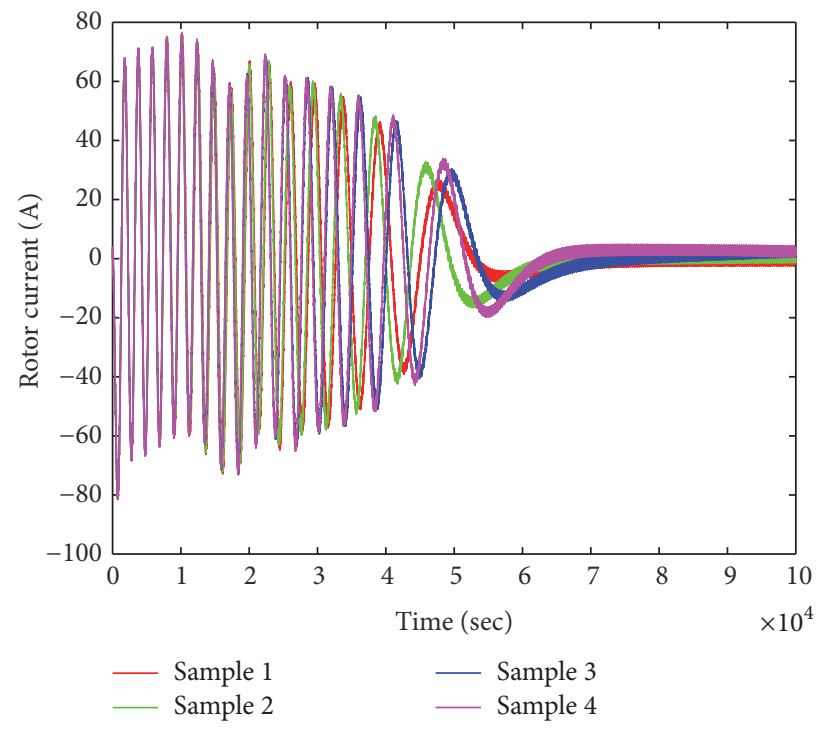

(b)

FIGURE 5: Rotor current comparison of at (a) no-load and (b) rotor lock full load.

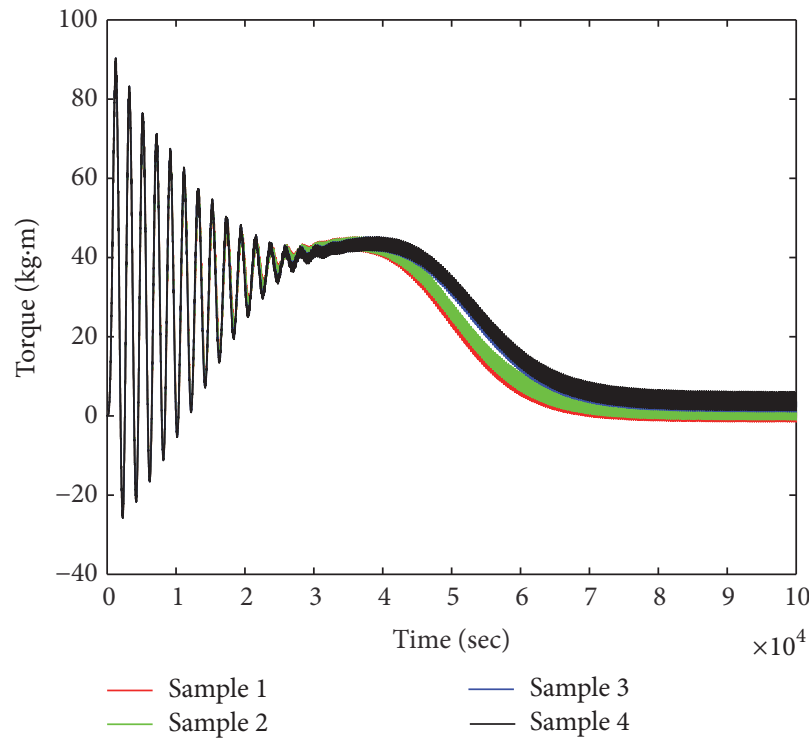

(a)

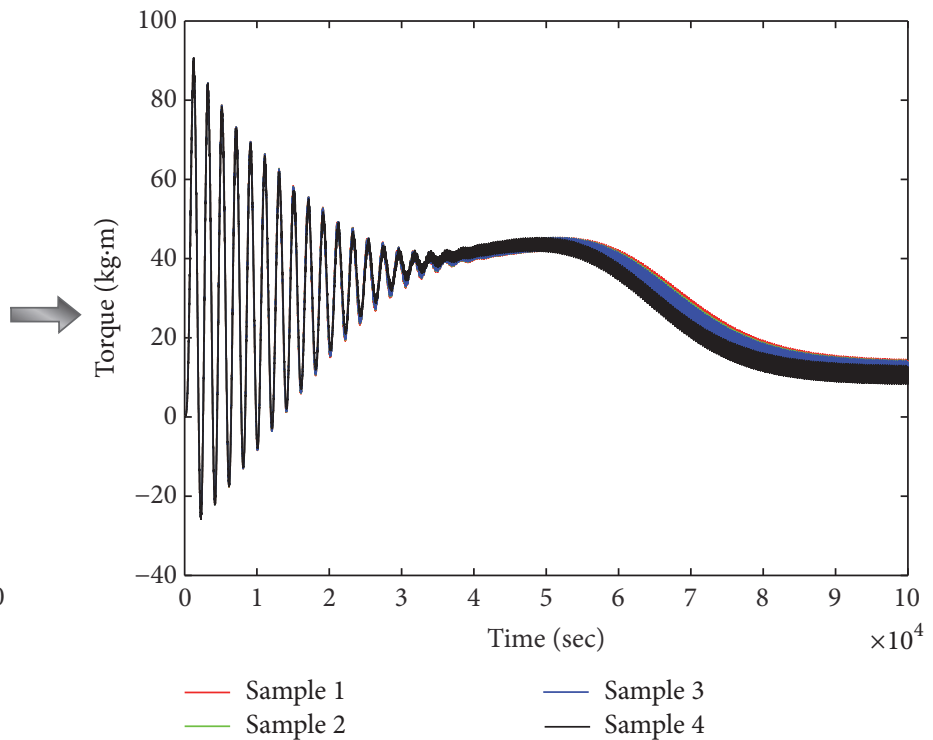

(b)

FIGURE 6: Rotor torque comparison of at (a) no-load and (b) rotor lock full load.

TABLE 3: Measured fault frequencies at various load condition.

\begin{tabular}{lccccc}
\hline Load condition & Sample number & Speed $(\mathrm{rpm})$ & Slip & RMS & Crest factor \\
\hline \multirow{4}{*}{ No load } & S1 & 1485 & 0.010 & 0.252 & 0.152 \\
& S2 & 1495 & 0.003 & 0.255 & 0.160 \\
& S3 & 1480 & 1488 & 0.013 & 0.285 \\
Full load & S4 & 1380 & 0.008 & 0.271 & 0.157 \\
& S1 & 1350 & 0.111 & 0.222 & 0.221 \\
& S2 & 1352 & 0.109 & 0.201 & 0.195 \\
& S3 & 1290 & 0.162 & 0.214 & 0.199 \\
\hline
\end{tabular}


TABLE 4: Description of the implemented ANN.

\begin{tabular}{ll}
\hline Tasks & Configuration of the implemented ANN \\
\hline Network type & Feed Forward Neural Network \\
Learning method & Back propagation \\
Training goal & 0.01 \\
Input data of each motor for each & The input is the one-dimensional matrix for the ANN of 4 inputs where all data in each sensing \\
experiment & point near motor are in a fault index \\
Number of hidden layer neurons & $\begin{array}{l}\text { Different architectures are applied with different neurons. For example, }[4 \times 3 \times 4],[4 \times 10 \times 4], \\
\text { and }[4 \times 15 \times 4](\text { see Figure 9) }\end{array}$ \\
Vector of classes for the target outputs & Numerical matrices refer to the fault with value 1 or 0 \\
\hline
\end{tabular}

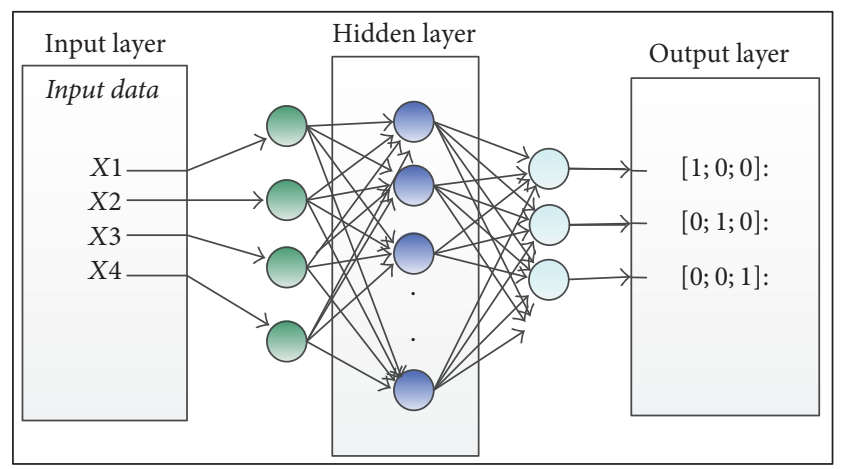

FIgURE 7: ANN classification network architecture.

the development of case study, initially, single motor feature vectors are trained through proposed network to observe the performance. The proposed architecture of ANN for motor is presented in Figure 7.

Output layer provides the condition of the motor. It consists of four neuron nodes and activation function logsig is used for each output [21]. Three types of architectures ([4 $\times$ $3 \times 3],[4 \times 10 \times 3]$, and $[4 \times 15 \times 3])$ are tested to achieve the required output in suitable timeframe. For the target output, a vector classes are formulated as follows:

(i) $[1 ; 0 ; 0]$ : For healthy/faulty condition

(ii) $[0 ; 1 ; 0]$ : For broken rotor bar fault

(iii) $[0 ; 0 ; 1]$ : For unknown fault.

All feature values were stored in Mat files and assigned these values with the motor. The MATLAB scripts were used to merge all the features and generate the entire set of training data range for the validation and testing process in both fault and nonfault cases. Figure 8 presents the inside architecture of each neural network for motor.

6.1. Model Setup and Training. Once the use of the neural network for the nonlinear modelling of a system has been established, certain neural network particulars have to be considered and decided upon, such as these precedents:

(i) Total number of hidden layers and their neurons

(ii) Transfer functions in neurons

(iii) Error criteria

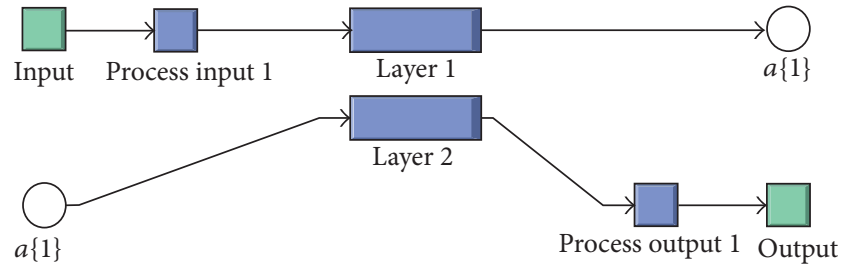

FIGURE 8: Internal architecture of Neural Network.

(iv) Training algorithm

(v) Stop criteria when output achieved

(vi) Initial values of the weights within all layers.

No attempt is made in this paper to describe all the above particulars in detail. The objective is rather to provide a general overview of the decisions one might face when designing an MLP. The brief configuration details of the neural network have been outlined as shown in Table 4 and Figure 9.

It can be seen in Table 5 that architecture $[4 \times 10 \times 4]$ has presented the good Mean Squared Error (MSE) performance in classification among all tested architectures. The reasonable numbers of epochs were used during the training process in suitable processing time to attain the necessitated precision, which demonstrates the good efficiency in the all tested architectures, along with the smallest number of error percentage. Figure 10 presents the training performance graph of the neural network architecture $[4 \times 10 \times 3]$, which achieved the justifiable performance result of the Neural Network testing.

After measuring the neural network testing performance of motor, the next step was to measure the classification confusion matrices for the diverse types of error that occurred during the training process and reduce them. To build the confusion matrix, sample feature data is provided into the neural network model (Figure 9). In model, the confusion matrix is holding the information about the comparison between predicted and targeted classification classes. Figure 11 shows the confusion matrices for the three process phases of training, testing and validation of motor current samples respectively. Four targeted and predicted classes (horizontal and vertical) were delineated to compare all sample feature data sets. In the case of successful classification of a targeted class trial, the diagonal cells are shown in green. 
TABLE 5: Performance of different architectures for classification.

\begin{tabular}{|c|c|c|c|c|c|}
\hline Architecture & Sample & MSE performance & Number of epochs & Accuracy (\%) & Classification error \\
\hline \multirow{4}{*}[4\times3\times3]{} & $\mathrm{S} 1$ & $6.69 \times 10^{-3}$ & 70 & 92.5 & 7.5 \\
\hline & S2 & $6.19 \times 10^{-3}$ & 75 & 93.4 & 6.6 \\
\hline & S3 & $7.37 \times 10^{-3}$ & 67 & 92.6 & 7.4 \\
\hline & S4 & $8.01 \times 10^{-3}$ & 100 & 94.0 & 6.0 \\
\hline \multirow{4}{*}[4\times10\times3]{} & S1 & $8.49 \times 10^{-3}$ & 117 & 96.2 & 3.8 \\
\hline & S2 & $8.59 \times 10^{-3}$ & 125 & 96.3 & 3.7 \\
\hline & S3 & $8.72 \times 10^{-3}$ & 132 & 97.4 & 2.6 \\
\hline & S4 & $8.99 \times 10^{-3}$ & 131 & 97.1 & 2.9 \\
\hline \multirow{4}{*}[4\times15\times3]{} & S1 & $8.01 \times 10^{-3}$ & 329 & 94.1 & 5.9 \\
\hline & S2 & $6.23 \times 10^{-3}$ & 327 & 93.5 & 6.5 \\
\hline & S3 & $7.85 \times 10^{-3}$ & 346 & 83.1 & 16.9 \\
\hline & S4 & $8.56 \times 10^{-3}$ & 387 & 91.8 & 8.2 \\
\hline
\end{tabular}

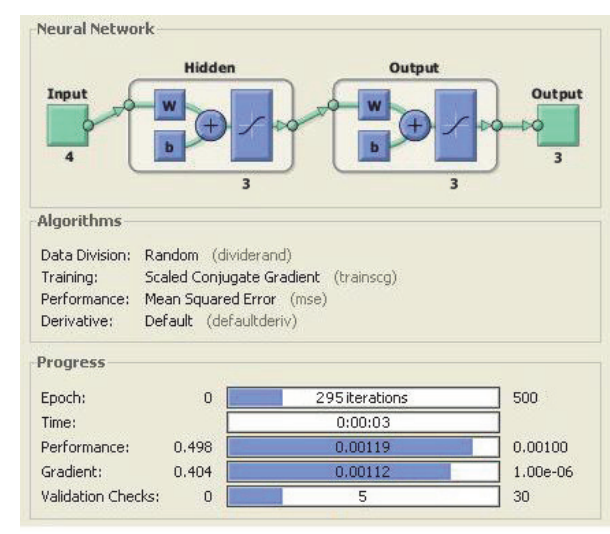

(a)

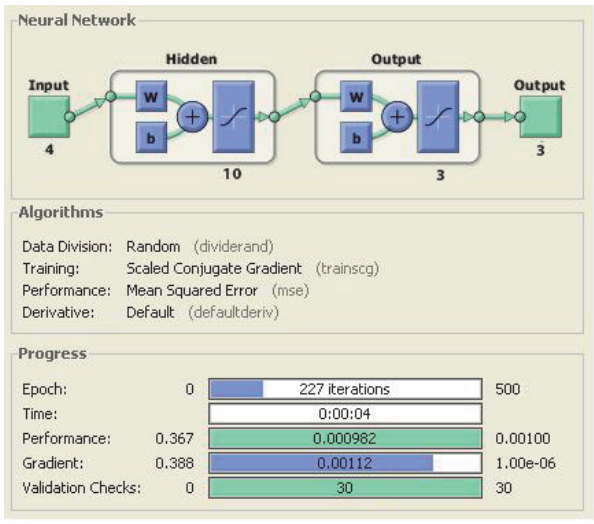

(b)

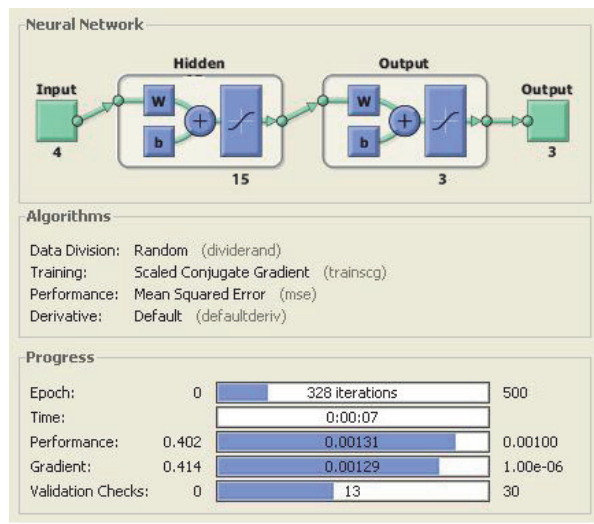

(c)

FIGURE 9: Overview of the different ANN architectures chosen for fault diagnosis. (a) $[4 \times 3 \times 3]$; (b) $[4 \times 10 \times 3]$; $(\mathrm{c})[4 \times 15 \times 3]$.

Each diagonal cell indicates the number of cases that have been classified correctly by the neural network, to identify feature condition, whether healthy or faulty for each motor. The cells in red colour point out the number of cases that have been erroneously classified by the ANN model or where the state of sample features were not clearly acknowledged. The blue cell indicates the overall percentage of tested cases that were classified correctly in green and the vice-versa in red.
In case of sample 1, Figure 11 shows each class had maximum of 1200 testing trials that are already preset in model. In order to reading vertically, 947 trials were successfully classified as class 1 . A total of 13 trials were wrongly classified as class 2, and 35 trials were wrongly classified in class 3 . In class 4 , a total of 5 trials were only erroneously classified due to complex nature of signal and mixing of different features in data sets. When the confusion matrix is read horizontally, 

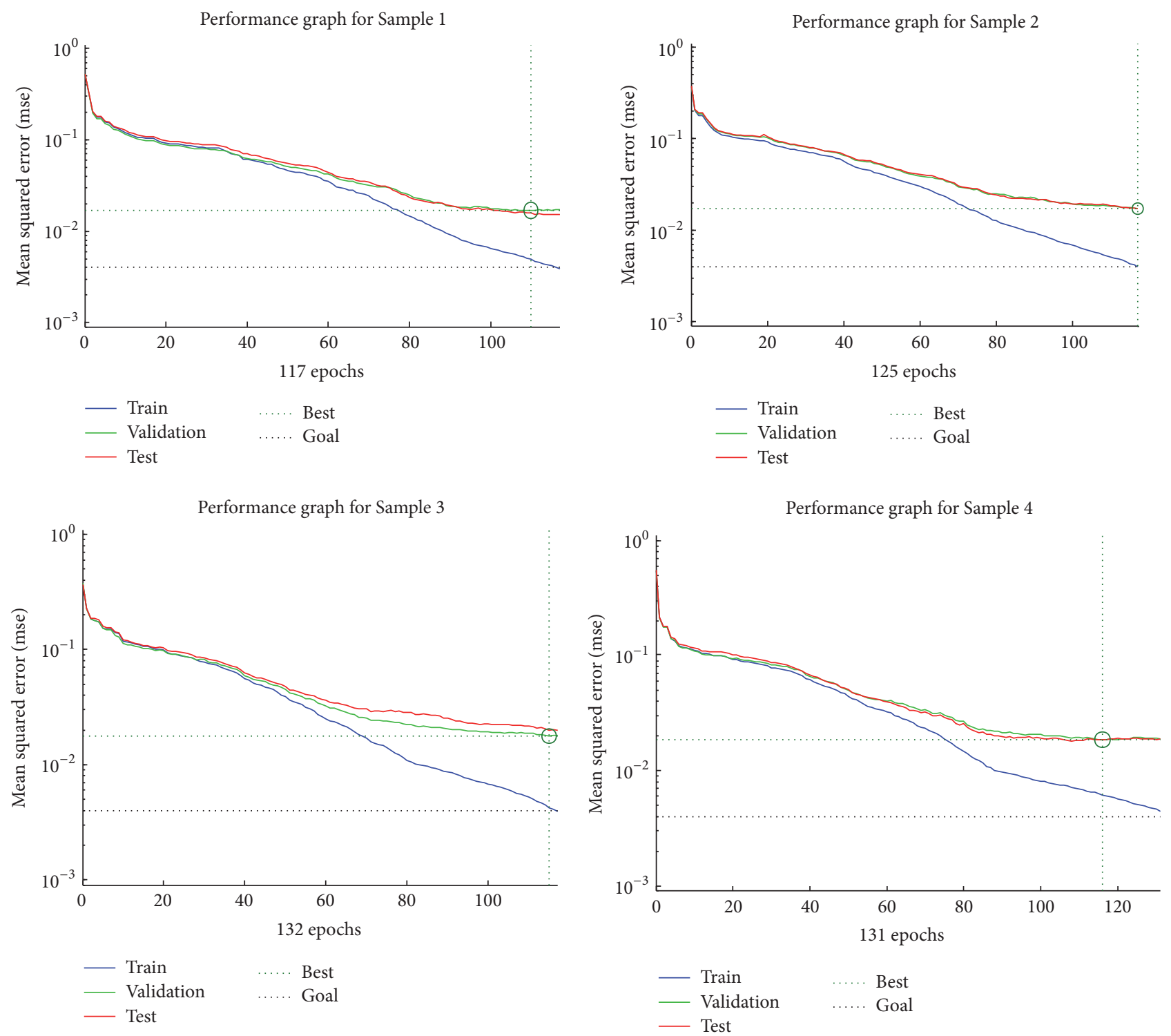

FIGURE 10: Performance graphs samples using $[4 \times 10 \times 4]$ neural network architecture.

24 trials of class 2, 30 trials of class 3 and 9 trials of class 4 were wrongly classified in model. Finally, last row (in grey colour) shows the successful classification percentage rate of each target class. The totals of 3952 testing trials were classified and the final performance rate of success was 96.2 percent. Only a 3.8 per cent error-rate occurred, which is quite an efficient and reasonable rate in industrial motor fault diagnosis process. In the confusion matrices of other samples, the success rate is also acceptable and shows the better performance in suggested NN architectural model.

It can be seen that chosen neural network architecture [4 $\times 10 \times 3$ ] demonstrated a reasonable and acceptable accuracy was achieved in fault diagnosis in the feature vector, ranging from 96 to 97 percent. This reflects the performance efficiency of the ANN algorithm, in reducing the level of uncertainty in decision-making and validity of sample trained data. To compare the confusion matrix performance of the proposed methodology and architecture, some work is needed on other motor faults.

\section{Conclusion and Future Directions}

A supervised neural network architectural model is presented to show the network efficiency for the fault diagnosis process in operating motor within industrial environment at two levels of loads torque. Significant features are chosen to identify the BRB fault that provides the information on motor operating condition through the back propagation algorithm and explained the reason for selection among several features. An industrial experimental setup has been used to extract the sample data at different load torque condition in every five seconds of interval of time. Noise factor is also considered to create the complexity in identification of faults symptoms. The simulated results showed that the precise and generality 

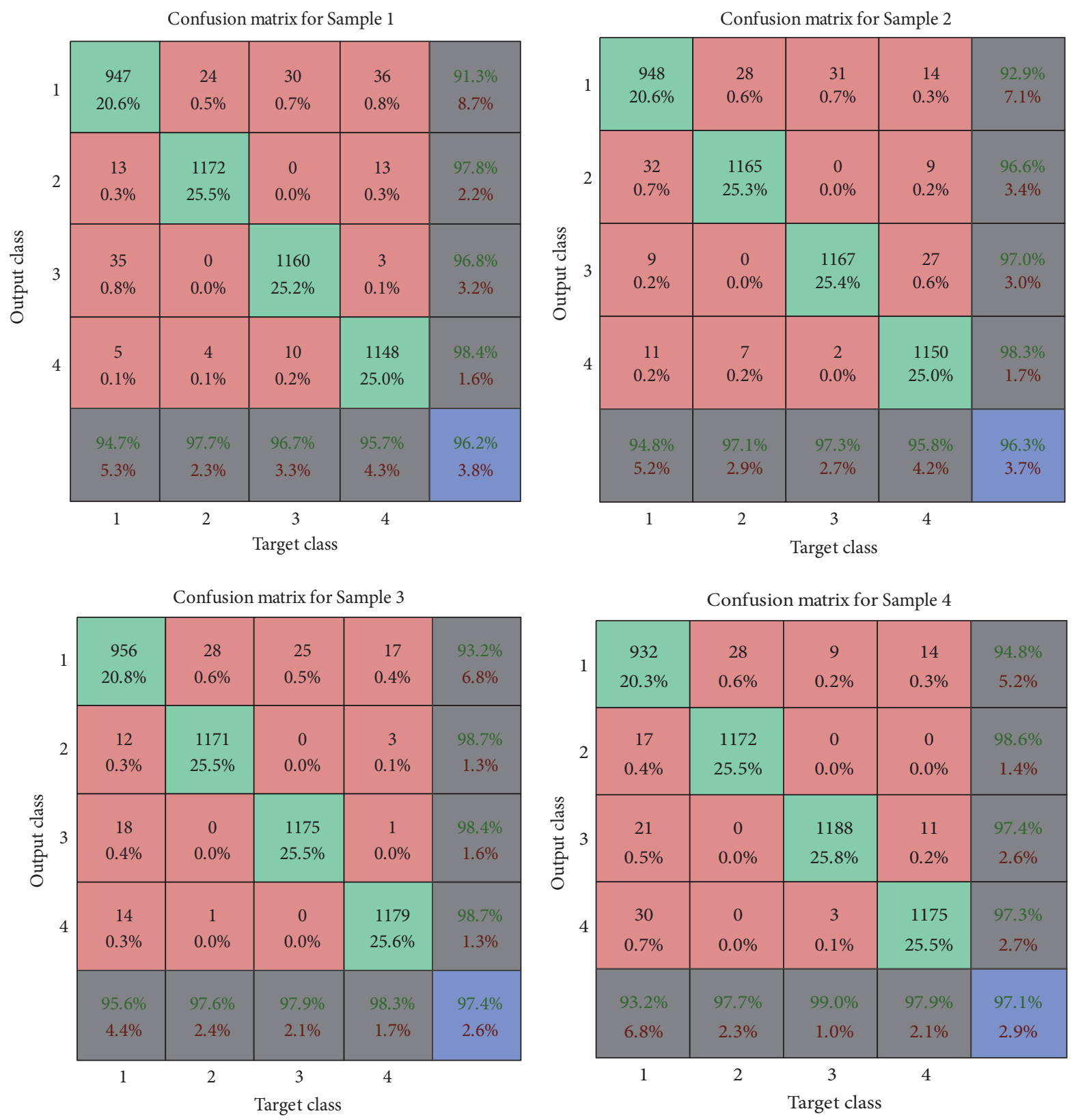

FIGURE 11: Confusion matrices of four samples of data using targeted and output classes.

behaviour of motor at different load levels is obtained to diagnose the motor fault in operating condition. To improve the MSE performance accuracy rate, three types of neural network architectures $[4 \times 10 \times 3]$ demonstrated a reasonable and acceptable accuracy was achieved in BRB fault diagnosis in the feature vector, ranging from 96 to 97 percent.

Future development of this research would be included the expansion of motors environment that will be running in parallel with same power-line by using some other knowledge-level diagnosis based approaches for decision validity. The propagation of the faulty signal through the electric power-line will also be deal with manifesting into healthy signal and creating the complexity in decision-making about the multiple motor health simultaneously by using the Wireless Sensor Network.

\section{Competing Interests}

The authors declare that there are no competing interests regarding the publication of this paper.

\section{Acknowledgments}

The authors would like to thank Ministry of Science and Innovation, New Zealand, for funding the research and AUT for hosting and implementing the project. 


\section{References}

[1] Y. Cai, Y. He, A. Li, J. Zhao, and T. Wang, "Application of wavelet to gearbox vibration signals for fault detection," in Proceedings of the 2nd International Conference on Advanced Computer Control (ICACC '10), vol. 4, pp. 441-444, March 2010.

[2] T. V. Nguyen and H. W. Belson, A System Approach to Machinery Condition Monitoring and Diagnosis, Lockheed Martin Information System, 2003.

[3] Handbook of Multisensory Data Fusion: Theory and Practice, Electrical Engineering \& Applied Signal Processing, CRC Press, 2nd edition, 2008.

[4] D. Logan and J. Mathew, "Using the correlation dimension for vibration fault diagnosis of rolling element bearings-I. Basic concepts," Mechanical Systems and Signal Processing, vol. 10, no. 3, pp. 241-250, 1996.

[5] G. Vachtsevanos, F. Lewis, M. Roemer, A. Hess, and B. Wu, "Frontmatter," in Intelligent Fault Diagnosis and Prognosis for Engineering Systems, John Wiley \& Sons, Hoboken, NJ, USA, 2007.

[6] M. R. W. Group, "Report of large motor reliability survey of industrial and commercial installations, part II," IEEE Transactions on Industry Applications, no. 21, pp. 865-872, 1985.

[7] R. P. Panadero, J. P. Llinares, V. C. Alarcon, and M. P. Sánchez, "Review diagnosis methods of induction electrical machines based on steady state current," in Proceedings of the 11th Spanish Portuguese Conference on Electrical Engineering, 2009.

[8] K. V. Kumar, S. S. Kumar, B. Praveena, J. P. John, and J. E. Paul, "Soft computing based fault diagnosis," in Proceedings of the 2nd International Conference on Computing, Communication and Networking Technologies (ICCCNT '10), pp. 1-7, Karur, India, July 2010.

[9] S. Nandi, H. A. Toliyat, and X. Li, "Condition monitoring and fault diagnosis of electrical motors-a review," IEEE Transactions on Energy Conversion, vol. 20, no. 4, pp. 719-729, 2005.

[10] A. Naha, A. K. Samanta, A. Routray, and A. K. Deb, "A method for detecting half-broken rotor bar in lightly loaded induction motors using current," IEEE Transactions on Instrumentation and Measurement, vol. 65, no. 7, pp. 1614-1625, 2016.

[11] V. Climente-Alarcon, J. A. Antonino-Daviu, E. G. Strangas, and M. Riera-Guasp, "Rotor-bar breakage mechanism and prognosis in an induction motor," IEEE Transactions on Industrial Electronics, vol. 62, no. 3, pp. 1814-1825, 2015.

[12] R. A. Patel and B. Bhalja, "Induction motor rotor fault detection using Artificial Neural Network," in Proceedings of the International Conference on Energy Systems and Applications, pp. 4550, Pune, India, October 2015.

[13] J. Rangel-Magdaleno, H. Peregrina-Barreto, J. Ramirez-Cortes, R. Morales-Caporal, and I. Cruz-Vega, "Vibration analysis of partially damaged rotor bar in induction motor under different load condition using DWT,' Shock and Vibration, vol. 2016, Article ID 3530464, 11 pages, 2016.

[14] S. Sridhar, K. Uma Rao, and S. Jade, "Identification of broken rotor bar fault and degree of loading in induction motor using neuro-wavelets," in Proceedings of the IEEE Region 10 Conference (TENCON '15), pp. 1-5, IEEE, Macao, China, November 2015.

[15] S. S. Refaat, H. Abu-Rub, M. S. Saad, and A. Iqbal, "Open and closed-loop motor control system with incipient broken rotor bar fault detection using current signature," in Proceedings of the 40th Annual Conference of the IEEE Industrial Electronics Society (IECON '14), pp. 774-780, Dallas, Tex, USA, November 2014.
[16] E. M. T. Eldin, H. R. Emara, E. M. Aboul-Zahab, and S. S. Refaat, "Monitoring and diagnosis of external faults in three phase induction motors using artificial neural network," in Proceedings of the IEEE Power Engineering Society General Meeting, pp. 1-7, Tampa, Fla, USA, June 2007.

[17] H. Arabac1 and O. Bilgin, "Diagnosis of broken rotor bar faults by using frequency spectrum of stator current envelope," in Proceedings of the 20th International Conference on Electrical Machines (ICEM '12), pp. 1643-1646, Marseille, France, September 2012 .

[18] J. Huang, X. Cao, and W. Li, "Fault diagnosis of rotating machinery based on empirical mode decomposition and fractal feature parameter classification," Key Engineering Materials, vol. 439-440, pp. 658-663, 2010.

[19] S. Altaf, A. Al-Anbuky, and H. G. Hosseini, "Fault signal propagation in a network of distributed motors," in Proceedings of the IEEE 8th International Power Engineering and Optimization Conference (PEOCO '14), pp. 59-63, IEEE, Langkawi, Malaysia, March 2014.

[20] S. Altaf, A. Al-Anbuky, and H. GholamHosseini, "Fault diagnosis in a distributed motor network using Artificial Neural Network," in Proceedings of the International Symposium on Power Electronics, Electrical Drives, Automation and Motion (SPEEDAM '14), pp. 190-197, IEEE, Ischia, Italy, June 2014.

[21] J. Huang, M. Wang, and W. Li, "A new decision-level fusion method for fault diagnosis," International Journal of Digital Content Technology and its Applications, vol. 5, no. 3, pp. 136142, 2011. 


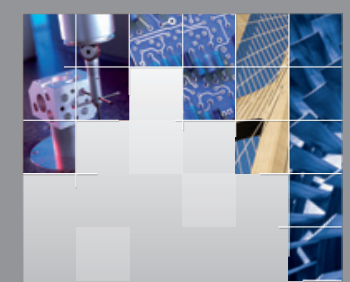

\section{Enfincering}
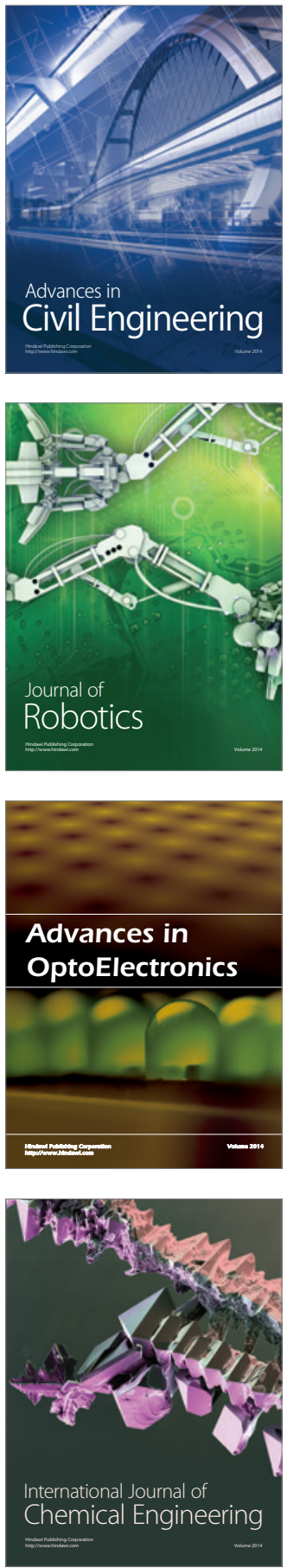

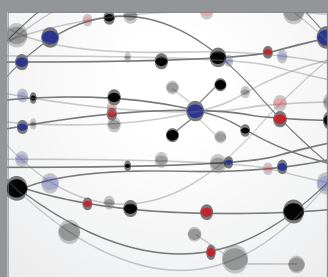

The Scientific World Journal

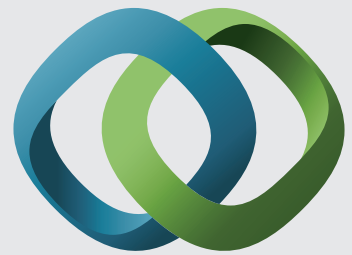

\section{Hindawi}

Submit your manuscripts at

https://www.hindawi.com
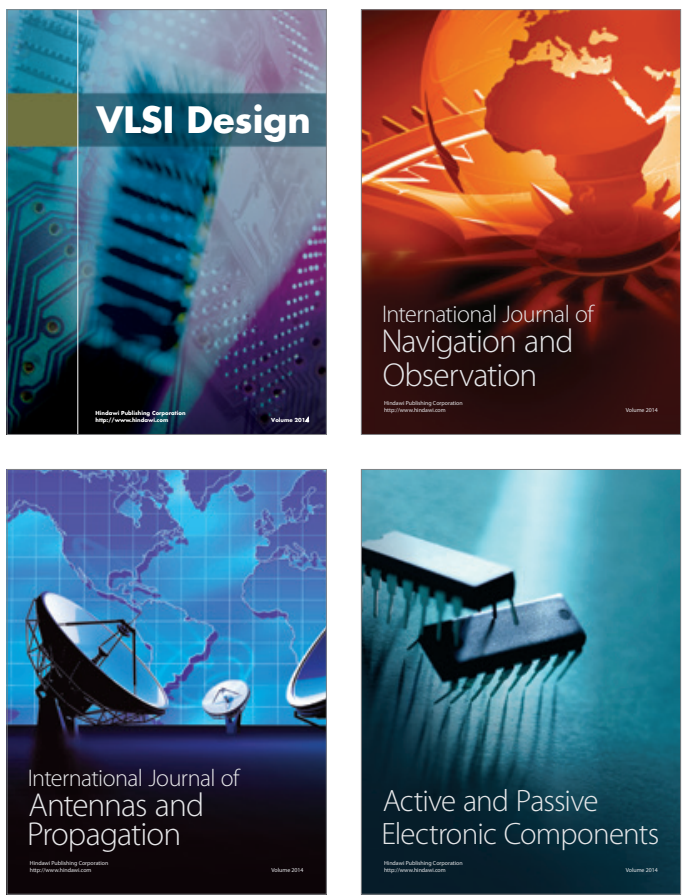
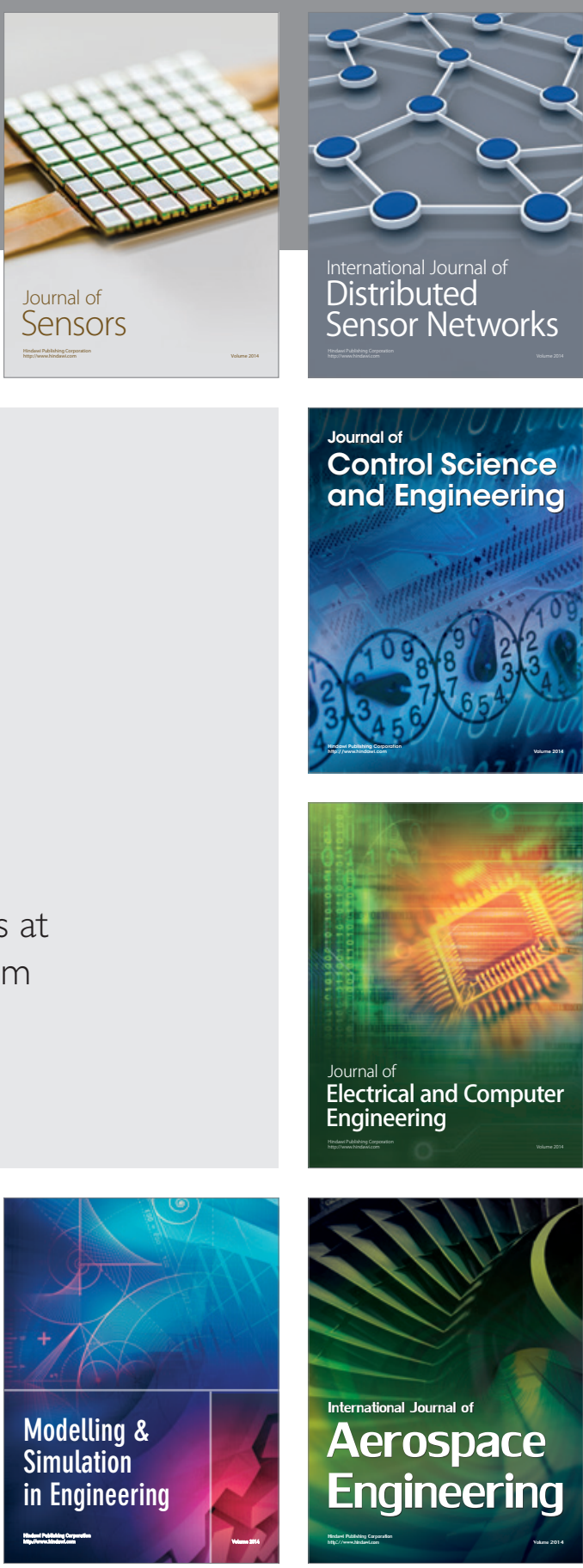

International Journal of

Distributed

Sensor Networks

$-$

Joumal of

Control Science

and Engineering
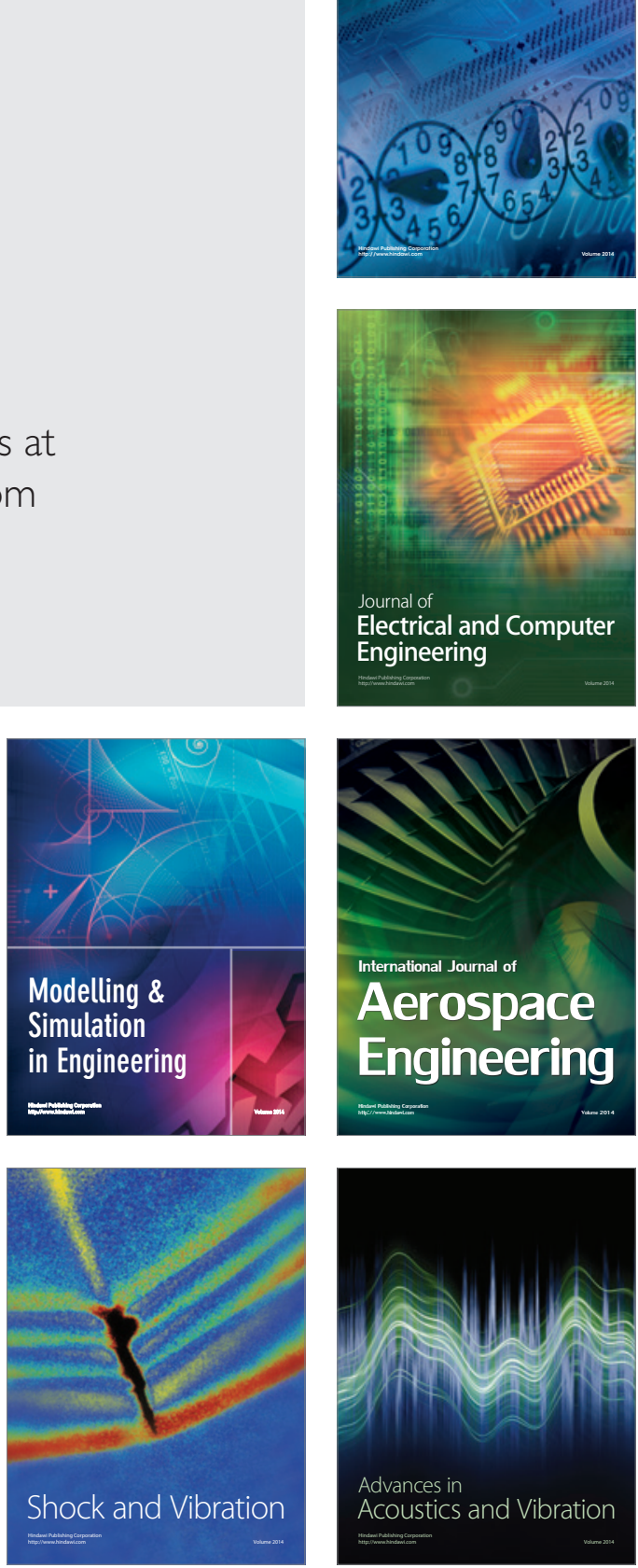